\title{
Short-term outcomes of laparoscopic colorectal resection in psychiatric patients
}

\author{
Ken Yuu ${ }^{1}$, Kazuhito Yajima ${ }^{2}$, Masanori Tada ${ }^{3}$, Nasry Baongoc ${ }^{3}$, Kurumi Tsuchihashi ${ }^{1}$, Masao Ogawa', Masayasu \\ Kawasaki ${ }^{1}$, Masao Kameyama ${ }^{1}$
}

${ }^{1}$ Department of Surgery, Bell Land General Hospital, Sakai, Osaka 599-8247, Japan.

${ }^{2}$ Department of Surgery, Sainokuni Higashiomiya Medical Center, Saitama-shi, Saitama 331-8577, Japan.

${ }^{3}$ Department of Surgery, Tokyo Metropolitan Matsuzawa Hospital, Setagaya-ku, Tokyo 156-0057, Japan.

Correspondence to: Dr. Ken Yuu, Department of Surgery, Bell Land General Hospital, 500-3, Higashiyama, Naka-ku, Sakai, Osaka 599-8247, Japan. E-mail: k_yuu@seichokai.or.jp

How to cite this article: Yuu K, Yajima K, Tada M, Baongoc N, Tsuchihashi K, Ogawa M, Kawasaki M, Kameyama M. Short-term outcomes of laparoscopic colorectal resection in psychiatric patients. Mini-invasive Surg 2017;1:173-9.

\section{Article history: \\ Received: 14 Aug 2017 \\ First Decision: 21 Sep 2017 \\ Revised: 6 Oct 2017 \\ Accepted: 12 Oct 2017 \\ Published: 28 Dec 2017 \\ Key words: \\ Laparoscopic surgery, \\ psychotic disorder, \\ colorectal cancer}

\begin{abstract}
Aim: To investigate the short-term outcomes of laparoscopic colorectal resection compared with open surgery in psychiatric patients with colorectal cancer. Methods: The authors retrospectively reviewed the medical records of 31 consecutive patients who underwent open surgery (OS) or laparoscopic surgery (LS) for colorectal cancer between April 2013 and September 2015. All patients were involuntarily admitted to the hospital, because of anosodiaphoria. The clinicopathological characteristics, intraoperative outcomes, and postoperative data of the two groups were analyzed. Categorical data were compared using the $\chi^{2}$ test or Fisher exact test, as appropriate. Continuous variables were compared using the Student $t$ test or Mann-Whitney $U$ test, as appropriate. Statistical analyses were performed using the statistical software program, SPSS, version 22 (SPSS Japan, Tokyo). $P$-values $<0.05$ were considered statistically significant. Results: Sixteenpatients underwent LS, and 15 underwent OS. Blood loss was lower in the LS group than in the OS group $(P=0.001)$. LS was associated with the earlier resumption of psychiatric drug treatment $(P<0.001)$ and a shorter hospital stay $(P=0.021)$ compared with OS. Conclusion: Laparoscopic colorectal surgery is safe for psychiatric patients. The main advantages of LS include a shorter washout period and reduced hospital stay.
\end{abstract}

\section{INTRODUCTION}

Laparoscopic colectomy has become accepted as an alternative to conventional open surgery (OS) for treating colon cancer because it results in earlier recovery. For patients with colorectal cancer, the benefits of laparoscopic surgery (LS) include fewer postoperative complications, shorter periods of hospitalization, and a more rapid recovery ${ }^{[1,2]}$. Therefore, LS may achieve better short-term outcomes than OS in psychiatric patients with colorectal cancer.

Patients with serious mental illnesses are at a significantly increased risk of death from cardiovascular, gastrointestinal, or respiratory disease ${ }^{[3-5]}$. Furthermore, clinical data suggest that patients with such conditions exhibit worse surgical outcomes than other patients ${ }^{\left[{ }^{[6]}\right.}$.

Patients with psychiatric disorders who develop cancer

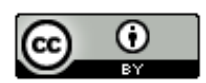

and reproduction identical terms.

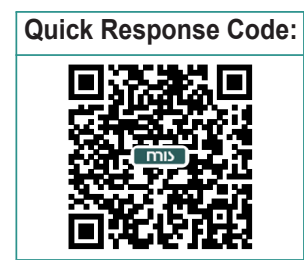


often present with complicated medical and psychiatric problems ${ }^{[7]}$. Such patients have difficulty coping with the diagnosis and treatment of cancer, and they may ignore warning signs and symptoms of the disease because of their cognitive impairment ${ }^{[8]}$, which can result in riskier treatments having to be performed.

Herein, we report the surgical outcomes of psychiatric patients with colorectal cancer. The aim of this study was to evaluate the surgical feasibility and safety of LS. Thus, we compared the operative, pathological, and functional outcomes of LS with those of OS in this patient population.

\section{METHODS}

A retrospective analysis was performed using a colorectal cancer database. Information in the database was prospectively collected between April 2013 and September 2015 at the Tokyo Metropolitan Matsuzawa Hospital. Thirty-one patients underwent LS or OS for primary colorectal cancer. We investigated the shortterm outcomes of laparoscopic colorectal resection compared with OS in psychiatric patients with colorectal cancer. All patients examined in this study were eligible for either approach. OS was performed between April 2013 and March 2014, and LS was started in April 2014. The exclusion criterion for LS was acute surgery due to perforative peritonitis. No patients were excluded from the present analysis because palliative surgery was performed or advanced disease was present. Informed consent was obtained from all patients or their relatives or guardians. This study was approved by the institutional review board of Tokyo Metropolitan Matsuzawa Hospital (approval number: 28).

Preoperative evaluations included colonoscopy with biopsy; chest, abdominal, and pelvic computed tomography examinations; and magnetic resonance imaging. Patients were staged using the Japanese Society for Cancer of the Colon and Rectum (eighth edition ${ }^{[9]}$. An American Society of Anesthesiologists (ASA) grade was assigned to each patient by an anesthetist preoperatively.

All patients were involuntarily admitted to the hospital because of anosodiaphoria. They were diagnosed as having colorectal cancer, but it was difficult to admit them to a general hospital for treatment other than psychiatric care. Tokyo Metropolitan Matsuzawa Hospital is one of the biggest hospitals in Tokyo that has a surgical and psychiatry department.

\section{Psychiatric disorders}

Details of the patients' psychiatric disorders are specified in Table 1. Schizophrenia $(n=17)$ and dementing disorders $(n=6)$ were overrepresented. The median disease period of all psychiatric disorders was 24 years (range: 0-58 years). Twenty-four patients had received psychotropic drugs orally, including at least one major psychotropic drug (a functional category of neuroleptic drugs that are helpful in treating psychosis and have the capacity to ameliorate thought disorders), and 19 had received minor psychotropic drugs (a drug that reduces anxiety). Psychotropic drugs include benzodiazepine derivatives and a few less widely used non-benzodiazepines, such as meprobamate and hydroxyzine hydrochloride). Seven patients had received epileptic agents, and 5 had taken donepezil. All patients had received at least one psychiatric medication. All patients were involuntarily admitted to the hospital; i.e. they were subjected to hospitalization for medical care and protection under Sections 1, 3, and 4 of the Mental Health and Welfare Act in Japan ${ }^{[10]}$. At our hospital, patients with psychiatric problems can be referred to psychiatrists at any time postoperatively, and 24-h psychiatric care is available, which enabled us to manage the study subjects in an unlocked surgical ward.

\section{Surgical technique}

No patients underwent mechanical and chemical bowel preparation. All laparoscopic and open procedures were performed by a single gastrointestinal surgical team. Laparoscopic procedures started to be performed in April 2014. The exclusion criterion for LS was acute surgery due to perforative peritonitis. All patients were operated on under general anesthesia. All laparoscopic and open procedures were performed according to the relevant guidelines ${ }^{[11]}$, and the extent of the resection was the same in both groups. All laparoscopic procedures were performed by the same surgeon, who was a proven expert, as defined by the Japan Society for Endoscopic Surgery. Conversion from LS to OS was allowed at the surgeon's discretion to ensure the patient's safety or because of technical difficulties, the presence of associated conditions, or findings of advanced disease or inadequate oncological margins. Reconstruction was performed with a hand-sewn straight colonic anastomosis or stapled anastomosis. A temporary ileostomy was created in selected patients. The same postoperative care was provided in all patients.

\section{Outcome assessment}

Postoperatively, the bowel motility recovery time, washout period, and length of the hospitalization period were assessed. The washout period was defined as the duration of days between the surgery and start of psychiatric medicine postoperatively. Postoperative 
Table 1: Psychiatric disorder of 31 patients

\begin{tabular}{|c|c|c|c|}
\hline Characteristics & LS $(n=16)$ & OS $(n=15)$ & $P$-value \\
\hline Disorders & & & 0.461 \\
\hline Schizophrenia & $9(56.3 \%)$ & $8(53.3 \%)$ & \\
\hline Dementing disorder & $4(25.0 \%)$ & $2(13.3 \%)$ & \\
\hline Manic-depressive illness & $0(0 \%)$ & $2(13.3 \%)$ & \\
\hline Drug addiction & $1(6.3 \%)$ & $0(0 \%)$ & \\
\hline Alcoholism & $2(12.5 \%)$ & $1(6.7 \%)$ & \\
\hline Bipolar affective disorder & $0(0 \%)$ & $1(6.7 \%)$ & \\
\hline Growth ret & 0( & $1(6.7 \%)$ & \\
\hline Disease duration (years) (range) & 20 & $15(0-58)$ & 0.626 \\
\hline \multicolumn{4}{|l|}{ Internal medicine } \\
\hline Major tranquilizer & $12(75.0 \%)$ & $12(80.0 \%)$ & 0.739 \\
\hline uilizer & $11(68.8 \%)$ & 8( & 0.379 \\
\hline Antiepileptic & $5(26.7 \%)$ & $2(13.3 \%)$ & 0.233 \\
\hline Anti-dementia drug & $3(18.8 \%)$ & $2(13.3 \%)$ & 0.982 \\
\hline Others & $4(25.0 \%)$ & $4(26.7 \%)$ & 0.916 \\
\hline More than two major tranquilizer & $6(37.5 \%)$ & $5(23.3 \%)$ & 0.809 \\
\hline $\begin{array}{l}\text { Kind of hospitalization } \\
\text { Involuntary admission" }\end{array}$ & $16(100 \%)$ & $15(100 \%)$ & - \\
\hline
\end{tabular}

*Value are the median (range); "hospitalization for medical care and protection under (section 1, 3, 4) of Mental Health and Welfare for Act in Japan

morbidity was defined as a complication that occurred within 30 days of the operation, and it was stratified as recommended by the Clavien-Dindo classification ${ }^{[12]}$. All pathological specimens were examined to determine the extent of the microscopic surgical margins.

\section{Statistical analysis}

Basic clinical characteristics, operative outcomes, and pathological results of the two groups were compared. Categorical data were compared using the $X^{2}$ test or Fisher exact test, as appropriate. Continuous variables were compared using the Student $t$ test or MannWhitney $U$ test, as appropriate. Statistical analyses were performed using the statistical software SPSS, version 22 (SPSS Japan, Tokyo, Japan). $P$-values < 0.05 were considered statistically significant.

\section{RESULTS}

\section{Patients' characteristics}

There were 16 patients in the LS group and 15 in the OS group. Demographic characteristics of both groups are shown in Table 2. No significant differences were observed between the two groups with respect to age, gender, body mass index, ASA score, or clinical stage (staging was performed before the surgical resection). The clinical and pathological stage was defined by the Japanese Classification of Colorectal Carcinoma, July 2013 (eighth edition) ${ }^{[9]}$.

\section{Perioperative outcomes}

Patients' intraoperative and postoperative data are listed in Table 3. Estimated blood loss was lower in the LS group than in the OS group $(P=0.001)$, and intraoperative transfusions were required less often in
Table 2: Clinicopathological characteristics

\begin{tabular}{|c|c|c|c|}
\hline Characteristics & LS $(n=16)$ & OS $(n=15)$ & $P$-value \\
\hline Age at surgery (years) ${ }^{\star}$ & $67(48-89)$ & $75(53-91)$ & 0.318 \\
\hline $\begin{array}{l}\text { Gender }(n) \\
\text { Male } \\
\text { Female }\end{array}$ & $\begin{array}{c}5(31.3 \%) \\
11(68.8 \%)\end{array}$ & $\begin{array}{c}5(33.3 \%) \\
10(66.7 \%)\end{array}$ & 0.901 \\
\hline BMI $\left(\mathrm{kg} / \mathrm{m}^{2}\right)^{*}$ & $19.6(15.5-32.5)$ & $19.8(15.0-24.9)$ & 1.000 \\
\hline $\begin{array}{l}\text { ASA score }(n) \\
\text { I } \\
\text { II } \\
\text { III }\end{array}$ & $\begin{array}{c}2(12.5 \%) \\
11(68.8 \%) \\
3(18.8 \%)\end{array}$ & $\begin{array}{c}0(0 \%) \\
13(86.7 \%) \\
2(13.3 \%)\end{array}$ & 0.311 \\
\hline $\begin{array}{l}\text { Previous abdominal } \\
\text { surgery }\end{array}$ & $4(25.0 \%)$ & $3(20.0 \%)$ & 0.561 \\
\hline $\begin{array}{l}\text { Preoperative intestinal } \\
\text { obstruction }\end{array}$ & $2(12.5 \%)$ & $3(20.0 \%)$ & 0.884 \\
\hline $\begin{array}{l}\text { Tumor location } \\
\text { Ascending colon } \\
\text { Transverse colon } \\
\text { Descending colon } \\
\text { Sigmoid colon } \\
\text { Rectum }\end{array}$ & $\begin{array}{l}2(12.5 \%) \\
2(12.5 \%) \\
1(6.3 \%) \\
7(46.7 \%) \\
4(25.0 \%)\end{array}$ & $\begin{array}{c}5(33.3 \%) \\
2(13.3 \%) \\
0(0 \%) \\
4(26.7 \%) \\
4(26.7 \%)\end{array}$ & 0.127 \\
\hline $\begin{array}{l}\text { No. clinical T stage } \\
\text { T2 } \\
\text { T3 } \\
\text { T4 }\end{array}$ & $\begin{array}{l}3(18.8 \%) \\
5(31.3 \%) \\
8(50.0 \%)\end{array}$ & $\begin{array}{c}0(0 \%) \\
9(60.0 \%) \\
6(40.0 \%)\end{array}$ & 0.103 \\
\hline $\begin{array}{l}\text { No. clinical N stage } \\
\text { N0 } \\
\text { N1-3 }\end{array}$ & $\begin{array}{l}7(43.8 \%) \\
9(56.4 \%)\end{array}$ & $\begin{array}{c}5(33.3 \%) \\
10(66.7 \%)\end{array}$ & 0.563 \\
\hline $\begin{array}{l}\text { No. clinical stage } \\
\text { I } \\
\text { II } \\
\text { III } \\
\text { IV }\end{array}$ & $\begin{array}{l}1(6.3 \%) \\
5(31.3 \%) \\
7(43.8 \%) \\
3(18.8 \%)\end{array}$ & $\begin{array}{c}0(0 \%) \\
5(33.3 \%) \\
10(66.7 \%) \\
0(0 \%)\end{array}$ & 0.212 \\
\hline
\end{tabular}

*Value are the median (range). LS: laparoscopic surgery; OS: open surgery; ASA: American society of anesthesiologist; BMI: body mass index

the LS group than in the OS group $(P=0.015)$. Two patients were converted from LS to OS. One of these patients had direct invasion into the urinary bladder, whereas the other had direct invasion into the splenic vein.

Compared with the OS group, the washout period, frequency of early postoperative recovery, and length of the hospitalization period were all significantly improved in the LS group. One patient in the LS group and 2 patients in the OS group who died were excluded in the length of postoperative hospital stay analysis. All patients except one $(93.8 \%)$, who did not have a drain inserted postoperatively, were subjected to temporary physical restraint; therefore, none of the patients removed their abdominal drains by themselves. A drain was removed the day after the first defecation postoperatively.

No patients received epidural anesthesia. The LS group required less frequent additional analgesia postoperatively, albeit not significantly. Postoperative delirium developed in 7 patients of the OS group, and 4 of the LS group. Unscheduled intravenous 
Table 3: Intraoperative and postoperative outcomes

\begin{tabular}{|c|c|c|c|}
\hline Characteristics & LS $(n=16)$ & OS $(n=15)$ & $P$-value \\
\hline Operation time $(\mathrm{min})^{*}$ & $193.5(125-459)$ & $155(78-483)$ & 0.188 \\
\hline Blood loss $(\mathrm{mL})^{*}$ & $27(10-1907)$ & $330(34-2197)$ & 0.001 \\
\hline Intraoperative transfusion & $2(12.5 \%)$ & $8(53.3 \%)$ & 0.015 \\
\hline Type of resection & & & 0.171 \\
\hline Right hemicolectomy & $3(18.8 \%)$ & $6(40.0 \%)$ & \\
\hline Transverse colectomy & $1(6.3 \%)$ & $1(6.7 \%)$ & \\
\hline Left hemicolectomy & $1(6.3 \%)$ & $0(0 \%)$ & \\
\hline Sigmoidectomy & $7(43.8 \%)$ & $3(20.0 \%)$ & \\
\hline Low anterior resection & $3(18.8 \%)$ & $1(6.7 \%)$ & \\
\hline Abdominal perineal resection & $0(0 \%)$ & $3(20.0 \%)$ & \\
\hline Total pelvic exenteration & $1(6.3 \%)$ & $0(0 \%)$ & \\
\hline Colostomy & $0(0 \%)$ & $1(6.7 \%)$ & \\
\hline Conversion to open surgery & $2(12.5 \%)$ & - & - \\
\hline Stoma & $1(6.3 \%)$ & $4(26.7 \%)$ & 0.122 \\
\hline Duration of drainage (days) & $8(0-13)$ & $8(7-27)$ & 0.175 \\
\hline Physical restraint & $15(93.8 \%)$ & $15(100 \%)$ & 0.325 \\
\hline Physical restraint (days) & $19.5(0-96)$ & $28(9-141)$ & 0.110 \\
\hline Recovery of bowel motility (days) & $2(1-6)$ & $7(2-25)$ & $<0.001$ \\
\hline Washout period (days) & $2.5(1-6)$ & $8(4-35)$ & $<0.001$ \\
\hline Return to diet (days) & $5.5(2-12)$ & $9(7-39)$ & 0.155 \\
\hline Incidence of using additional analgesia, $n(\%)$ & $3(18.8 \%)$ & $6(40.0 \%)$ & 0.252 \\
\hline Unscheduled Intravenous injections of psychotropic drugs & $4(25.0 \%)$ & $7(46.7 \%)$ & 0.208 \\
\hline Duration of hospital stay after surgery (days) & $43(18-144)$ & $69(29-423)$ & 0.021 \\
\hline
\end{tabular}

*Value are the median (range). LS: laparoscopic surgery; OS: open surgery

injections of psychotropic drugs were required for these patients. Such injections usually involved haloperidol, levomepromazine maleate, or diazepam or a combination of these drugs, as determined by the attending psychiatrist. The proportion of patients who needed unscheduled intravenous injections of psychotropic drugs did not differ significantly between the OS and LS groups. Physical restraints were applied for median periods of 19.5 days in the LS group and 28 days in the OS group; the difference was not significant $(P=0.110)$.

\section{Complications}

Postoperative complications occurred in 6 patients $(37.5 \%)$ in the LS group and $9(60.0 \%)$ in the OS group $(P=0.318)$. Major complications, which were defined as those that were more severe than Clavien-Dindo class III, occurred in 2 patients in the LS group and 4 in the OS group. One patient died (of pulmonary embolism 2 days postoperatively) in the LS group, and 2 patients died (one died of sepsis due to anastomotic leakage 105 days postoperatively, and the other died of acute liver failure due to liver metastasis 80 days postoperatively) in the OS group. Mortality rates of the two groups did not differ significantly [Table 4].

\section{Pathological outcomes}

Table 5 shows the pathological outcomes of both groups. There were no significant differences between the groups with respect to the tumor size, histological grade, or final stage. Eighteen of 31 (58.1\%) patients were diagnosed as having stage III or IV disease. Four patients in the LS group had macroscopic residual tumors. Three of these patients had liver metastasis, and the other patient had para-aortic lymph node metastasis. No patients received adjuvant or postoperative chemotherapy.

In the OS group, 4 patients had macroscopic residual tumors. Three of these patients had peritoneal metastasis. The remaining patient underwent colostomy because of direct invasion of the sacrum.

\section{DISCUSSION}

The number of patients receiving medical treatment from psychiatrists in Japan has increased over the past three decades. Moreover, the number of elderly patients with psychiatric disorders has increased nine-fold during the same period. Psychiatric patients are not considered to be at greater risk of digestive disease that requires operation than the general population ${ }^{[13]}$. Therefore, Japanese surgeons are increasingly having to perform operation in psychiatric patients. We have performed various surgical procedures for digestive disease in patients with psychiatric disorders, and we started performing LS at the Tokyo Metropolitan Matsuzawa Hospital in 2014.

There have only been a few reports about digestive surgery in patients with psychiatric disorders, and none 
Table 4: Postoperative morbidity

\begin{tabular}{lccc}
\hline Characteristics & LS $(\boldsymbol{n}=\mathbf{1 6})$ & OS $(\boldsymbol{n}=\mathbf{1 5})$ & $\boldsymbol{P}$-value \\
\hline Grade of morbidity & & & 0.643 \\
Clavien-Dindo I-II & $4(25.0 \%)$ & $4(26.7 \%)$ & \\
Clavien-Dindo III-IV & $1(6.3 \%)$ & $2(13.3 \%)$ & \\
Clavien-Dindo V & $1(6.3 \%)$ & $2(13.3 \%)$ & \\
Postoperative morbidity & & & 0.802 \\
Anastomotic leakage & $2(12.5 \%)$ & $1(6.7 \%)$ & \\
Intra-abdominal infection & $0(0 \%)$ & $1(6.7 \%)$ & \\
Ileus & $0(0 \%)$ & $2(13.3 \%)$ & \\
Wound infection & $1(6.3 \%)$ & $1(6.7 \%)$ & \\
Others & $3(18.8 \%)$ & $3(20.0 \%)$ & \\
\hline
\end{tabular}

*Total number of patients who suffered from postoperative morbidity. LS: laparoscopic surgery; OS: open surgery

of these studies focused on the safety and feasibility of laparoscopic colorectal surgery in psychiatric patients. It has been suggested that the mortality rate of psychiatric patients is higher than that of comparable non-psychiatric populations ${ }^{[14]}$. In addition, it has been reported that the hospitalization of patients with schizophrenia for medical or surgical reasons doubles the odds of several types of adverse events compared with the risk of such events in non-schizophrenic patients $^{[6]}$. However, Bernstein and Offenbart[ ${ }^{[15]}$ found that although patients with cognitive impairments have a higher than average mortality rate after general operation, they exhibit a similar incidence of non-fatal complications than surgical patients as a whole, and their increased mortality is mainly due to delays in diagnosis and their inability to withstand the technical surgical complications.

In the present study, we obtained similar overall morbidity and mortality rates to those described in a previous report ${ }^{[13]}$. However, our morbidity rate was higher than that reported for non-psychiatric patients ${ }^{[16]}$. As noted in the Introduction section, individuals with schizophrenia have higher pain thresholds than patients without mental illness, and they have more cognitive deficits, disorganized thinking, and other functional difficulties. Thus, they may have an impaired ability to recognize and communicate potentially important medical symptoms; thus, they may present with more advanced disease, resulting in the need to use riskier treatments ${ }^{[17]}$. In the current study, $58.1 \%$ of patients were diagnosed as having stage III or IV disease.

Estimated blood loss was lower in the LS group than in the OS group. However, the operative time was similar in both groups. Our results differ from those of previous studies ${ }^{[16]}$, and this is possibly because our study included a small number of patients. There were no significant differences in morbidity or mortality between the LS and OS groups. Our findings confirm that laparoscopic colectomy for psychiatric patients are not associated with a significant increase in the overall
Table 5: Pathologic characteristics

\begin{tabular}{lccc}
\hline Characteristics & LS $(\boldsymbol{n}=16)$ & OS $(\boldsymbol{n}=15)$ & $\boldsymbol{P}$-value \\
\hline Tumor size $(\mathrm{mm})^{*}$ & $44.5(19-100)$ & $56.0(34-130)$ & 0.240 \\
Retrieved LN $(n)^{*}$ & $17(5-39)$ & $16(0-29)$ & 0.423 \\
Histological differentiation & & & 0.138 \\
Well & $9(56.3 \%)$ & $3(20.0 \%)$ & \\
Moderate & $7(43.8 \%)$ & $10(66.7 \%)$ & \\
Poorly & $0(0 \%)$ & $1(6.7 \%)$ & \\
Tage & & & 0.363 \\
I & $2(12.5 \%)$ & $0(0 \%)$ & \\
II & $6(37.5 \%)$ & $4(26.7 \%)$ & \\
III & $4(25.0 \%)$ & $7(46.7 \%)$ & \\
IV & $4(25.0 \%)$ & $3(20.0 \%)$ & \\
Residual tumor ${ }^{*}$ & & & 0.992 \\
R0 & $11(62.5 \%)$ & $10(66.7 \%)$ & \\
R1 & $1(6.3 \%)$ & $1(6.7 \%)$ & \\
R2 & $4(25.0 \%)$ & $4(26.7 \%)$ & \\
\hline
\end{tabular}

*Value are the median (range); ${ }^{*}$ R0: no residual tumor; LN: Iymph node; R1: microscopic residual tumor; R2: macroscopic residual tumor. LS: laparoscopic surgery; OS: open surgery

complication rate. In the present study, anastomotic leakage occurred more frequently in the LS group, but not significantly. Colostomy was performed in 4 patients ( 3 with sigmoid colon cancer and 1 with rectal cancer) in the OS group, which prevented anastomotic leakage, whereas in the LS group, colostomy was only required in 1 patient who underwent pelvic exenteration. Colostomy is a safe procedure, because it does not require anastomosis. However, it is difficult to ensure that the resultant stoma is treated appropriately in psychiatric patients. Thus, the presence of a stoma can increase the risk of longer physical restraint, and it can be difficult to provide adequate stoma care after the patient has left the hospital. Therefore, the quality of life of psychiatric patients may be compromised by a lack of stoma care; hence, clinicians should be wary of performing colostomy in psychiatric patients.

Two patients were converted to OS from LS. One patient had a huge tumor that directly invaded the urinary bladder and rectum. The other patient had a tumor embolism in his inferior mesenteric vein and splenic vein. Psychiatric patients usually have megacolon due to a long disease period and psychiatric medicine [Figure 1] ${ }^{[17]}$. No patient was converted to OS, owing to megacolon and other psychiatric reason. As mentioned previously, patients with mental illness may present with more advanced disease $^{[17]}$, resulting in anemia, obstruction, or infiltration of other organs. Advanced cancer may have made the surgical technique complex. For psychiatric patients, it was not difficult to continue and complete LS in the present study. Thus, LS should be performed by an expert who has passed an endoscopic surgical skill qualification system.

The hospitalization period was significantly shorter in the LS group than in the OS group $(P=0.021)$. LS may 


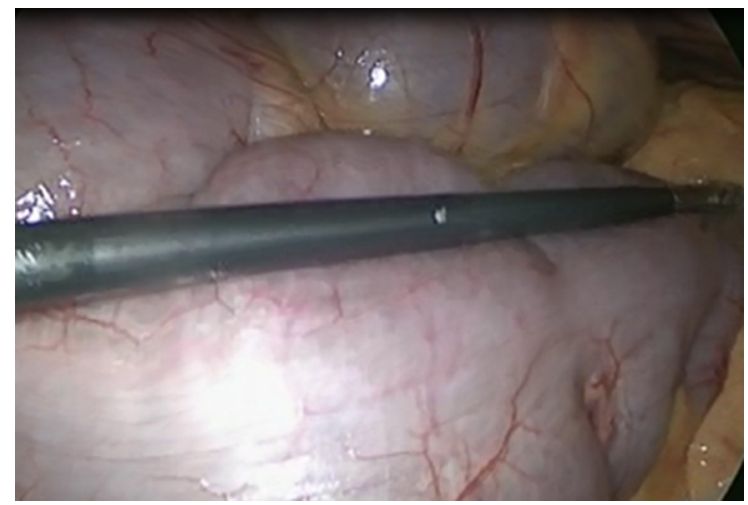

Figure 1: Almost all of patients had megacolon, owing to psychiatric medicine and some purgative medicine

have several short-term advantages compared with OS, e.g., it may contribute to reducing the hospitalization period. According to some reports, institutionalized patients tend to be hospitalized for longer, increasing the cost of surgical treatment for this population ${ }^{[6,18]}$. In our study, the median hospitalization period was 43 days in the LS group. One of the factors delaying the discharge of psychiatric patients is the difficulty that such patients have with adjusting to their environment. Patients with psychiatric problems cannot return to the psychiatric department or support facilities immediately after recovering from surgical stress. Collaboration with social workers may help reduce the hospitalization period by increasing clinicians' understanding of the optimal management methods for patients with psychiatric disorders.

The washout period was significantly shorter in the LS group than in the OS group. This suggests that psychiatric medications should be continued, if possible, during the perioperative period ${ }^{[6]}$. Until LS was introduced, patients at our hospital routinely received a nasogastric tube for a week postoperatively. The recovery of bowel motility is more difficult in psychiatric patients than in general patients. After starting LS, the nasogastric tube was removed immediately postoperatively ${ }^{[19]}$. The recovery of bowel motility postoperatively was assessed by a surgeon, auscultation, or other physical findings. Therefore, psychiatric medications were resumed as soon as possible. Increased rates of delirium and confusion in the postoperative period have been reported in psychiatric patients. In surgical, medical, and critically ill patients, delirium is associated with higher mortality, a longer hospital stay, and impairment at discharge ${ }^{[4,5]}$. Some studies have suggested that the postoperative development of confusion, delirium, or cognitive disorders may have important consequences for health care utilization and patient outcomes ${ }^{[20,21]}$. LS may lead to a more rapid recovery of intestinal peristalsis, even for psychiatric patients.

This study had several limitations. First, it had a retrospective study design that has the inherent weakness of being observational or non-experimental. Second, the follow-up period was too short to enable us to draw any conclusions regarding the long-term outcomes of the LS or OS group. However, by continuing to enroll and follow-up with patients, we hope to obtain more valuable information in the future. Third, the small number of subjects is the main limitation, as this may have affected the results of the parameters. The Tokyo Metropolitan Matsuzawa Hospital is one of the largest mental hospitals in Tokyo. Yet, there are about 20 patients who have colorectal cancer in 1 year, so it was difficult to include more psychiatric patients with cancer in our study.

In conclusion, in agreement with a previous study that found that patients with psychiatric disorders do not represent a high-risk group during surgical treatment ${ }^{[13]}$, we consider that such patients do not have worse outcomes after LS than after OS for colorectal cancer. Our study demonstrated that LS for psychiatric patients is safe, and it has comparable short-term outcomes and oncological results compared to OS. Psychiatric patients might not need special postoperative managements, excluding treatment for psychiatric problems. Further studies involving more patients and a longer follow-up period are needed to confirm the present study's results.

\section{DECLARATIONS}

\section{Acknowledgments}

We grateful thanks to Dr. Masahiko SAITO, the president of the metropolitan Matsuzawa Hospital and Dr. Naoto EGAWA who is the president of the Tokyo Metropolitan Hiroo Hospital. We would also like to thank Editage (www.editage.jp) for English language editing.

\section{Authors' contributions}

Concept, design, definition and intellectual content of this study: K. Yuu, M. Tada

Operated this series: K.Yuu, Nasry Baongoc, M Tada Analysis, statistical analysis of this study: K. Yajima, K. Yuu

Manuscript preparation: K. Yuu

Data acquisition: K Yuu, K Tsuchihashi, M Ogawa, M Kawasaki and M Kameyama

The paper was coauthored by Kazuhito Yajima, Masanori Tada, Nasry Baongoc, Kurumi Tsuchihashi, Masao Ogawa, Masayasu Kawasaki and Masao Kameyama. All authors' meets the authorship criteria detailed in the Authorship section of this guideline and 
all authors are in agreement with the content of the manuscript.

\section{Financial support and sponsorship None.}

\section{Conflictsofinterest}

There are no conflictsofinterest.

\section{Ethic approval}

We have approval of the research protocol by an institutional Review board. The study was reviewed and approved by the Tokyo Metropolitan Matsuzawa Hospital institutional review board. All study participants, or their legal guardian, provided informed written consent prior to their enrollment.

\section{REFERENCES}

1. Lacy AM, García-Valdecasas JC, Delgado S, Grande L, Fuster J, Tabet J, Ramos C, Piqué JM, Cifuentes A, Visa J. Postoperative complications of laparoscopic-assisted colectomy. Surg Endosc 1997;11:119-22.

2. Milsom JW, Böhm B, Hammerhofer KA, Fazio V, Steiger E, Elson P.A prospective, randomized trial comparing laparoscopic versus conventional techniques in colorectal cancer surgery: a preliminary report. J Am Coll Surg 1998;187:46-54; discussion 54-5.

3. Mortensen PB, Juel K. Mortality and causes of death in first admitted schizophrenic patients. Br J Psychiatry 1993;163:183-9.

4. Curkendall SM, Mo J, Glasser DB, Rose Stang M, Jones JK. Cardiovascular disease in patients with schizophrenia in Saskatchewan, Canada. J Clin Psychiatry 2004;65:715-20.

5. Kudoh A. Perioperative management for chronic schizophrenic patients. Anesth Analg 2005;101:1867-72.

6. Daumit GL, Pronovost PJ, Anthony CB, Guallar E, Steinwachs DM, Ford DE. Adverse events during medical and surgical hospitalizations for persons with schizophrenia. Arch Gen Psychiatry 2006;63:267-72.

7. Hashimoto N, Isaka N, Ishizawa Y, Mitsui T, Sasaki M. Surgical management of colorectal cancer in patients with psychiatric disorders. Surg Today 2009;39:393-8.

8. Inagaki T, Yasukawa R, Okazaki S, Yasuda H, Kawamukai T, Utani E, Hayashida M, Mizuno S, Miyaoka T, Shinno H, Horiguchi J. Factors disturbing treatment for cancer in patients with schizophrenia. Psychiatry Clin Neurosci 2006;60:327-31.

9. Japanese Classification of Colorectal Carcinoma (eighth edition). Japanese Society for Cancer of the Colon and Rectum 2013 July. p.
6-18. (in Japanese). Available from: http://www.kanehara-shuppan. co.jp. [Last accessed on 30 Oct 2017]

10. Japanese Association of Mental Health Services. Available from http://www.npo-jam.org/en/laws.html. [Last accessed on 30 Oct 2017]

11. Watanabe T, Itabashi M, Shimada Y, Tanaka S, Ito Y, Ajioka Y, Hamaguchi T, Hyodo I, Igarashi M, Ishida H, Ishiguro M, Kanemitsu Y, Kokudo N, Muro K, Ochiai A, Oguchi M, Ohkura Y, Saito Y, Sakai Y, Ueno H, Yoshino T, Fujimori T, Koinuma N, Morita T, Nishimura G, Sakata Y, Takahashi K, Takiuchi H, Tsuruta O, Yamaguchi T, Yoshida M, Yamaguchi N, Kotake K, Sugihara K; Japanese Society for Cancer of the Colon and Rectum. Japanese society for cancer of the colon and rectum (JSCCR) guidelines 2010 for the treatment of colorectal cancer. Int J Clin Oncol 2012;17:1-29.

12. Clavien PA, Barkun J, de Oliveira ML, Vauthey JN, Dindo D, Schulick RD, de Santibañes E, Pekolj J, Slankamenac K, Bassi C, Graf R, Vonlanthen R, Padbury R, Cameron JL, Makuuchi M. The ClavienDindo classification of surgical complications: five-year experience. Ann Surg 2009;250:187-96.

13. Aoyanagi N, Iizuka I, Watanabe M. Surgery for digestive malignancies in patients with psychiatric disorders. World J Surg 2007;31:2323-8.

14. Tsuang MT, Woolson RF. Mortality in patients with schizophrenia, mania, depression and surgical conditions. A comparison with general population mortality. Br J Psychiatry 1977;130:162-6.

15. Bernstein GM, Offenbartl SK. Adverse surgical outcomes among patients with cognitive impairments. Am Surg 1991;57:682-90.

16. Clinical Outcomes of Surgical Therapy Study Group, Nelson H, Sargent DJ, Wieand HS, Fleshman J, Anvari M, Stryker SJ, Beart RW Jr, Hellinger M, Flanagan R Jr, Peters W, Ota D. A comparison of laparoscopically assisted and open colectomy for colon cancer. $N$ Engl $J$ Med 2004;350:2050-9.

17. Adad SJ, Souza MA, Silva GB, Carmo Junior Jd, Godoy CA, Micheletti AM. Acquired non-Chagas megacolon associated with the use of psychiatric medication: case report and differential diagnosis with Chagas megacolon. Rev Soc Bras Med Trop 2008;41:293-5.

18. Kemp R, Hayward P, Applewhaite G, Everitt B, David A. Compliance therapy in psychotic patients: randomised controlled trial. $B M J$ 1996;312:345-9.

19. Lacy AM, García-Valdecasas JC, Delgado S, Castells A, Taurá P, Piqué JM, Visa J. Laparoscopy-assisted colectomy versus open colectomy for treatment of non-metastatic colon cancer: a randomised trial. Lancet 2002;359:2224-9.

20. Kudoh A, Katagai H, Takase H, Takazawa T. Effect of preoperative discontinuation of antipsychotics in schizophrenic patients on outcome during and after anaesthesia. Eur J Anaesthesiol 2004;21:414-6.

21. Milbrandt EB, Kersten A, Kong L, Weissfeld LA, Clermont G, Fink MP, Angus DC. Haloperidol use is associated with lower hospital mortality in mechanically ventilated patients. Crit Care Med 2005;33:226-9; discussion 263-5. 